DOI: http://dx.doi.org/10.21123/bsj.2017.14.1.0208

\title{
Stimulated Emission Cross Section in Xenon-Neon Lasers
}

\section{Gulalla Yaseen Bakr}

Physics Department, College of Education, Salahaddin University, Salahaddin, Iraq.

Received 10/7/2015

Accepted $22 / 2 / 2016$

(c) (i) $(9)$ This work is licensed under a Creative Commons Attribution-Non Commercial-No Derivatives 4.0 International License

\begin{abstract}
:
Gas Lasers are important tools that are used in variety purposes, for their low and (cw) output power. The aim of this study was to prepare a way to calculate an optimum stimulated emission cross-section in a gas laser containing a mixture of Xenon and Neon by (30\%-70\%). The process was a theoretical study of each gas in separate in terms of their physical properties as an active medium. The results of these calculations are logic and more convenient than other mixtures used before.
\end{abstract}

Key words: Gas-mixtures, Ionization and Excitation processes, Gas lasers

\section{Introduction:}

When gases are excited from their ground state several processes face this excitation, like collisions, scatting, absorption, transfer and ionization[1]. Out of these,in gases the process of emission is the matter of question in gas lasers. Since the most considerable process is the stimulated emission which results the process of amplification and power coupling. A laser has a resonator which contains usually an active medium to be amplified then[2] the consequent processes mentioned above own its special cross section. The algebraic sum of these give what is called the total cross section[3]. The two main portions of cross section are excitation and the momentum transfer cross sections. Studies prove that these are the back bone of both spontaneous and stimulated emission cross sections.

\section{Theory:}

The excitation cross section results through elastic atom-atom collision with cross section $\sigma_{e}=\frac{4 \pi}{k^{2}}(2 l+$ 1) $\sin ^{2} \delta_{l}(k)[4]$.

$f(r, k)=\delta_{l}(k) \cdot k$

Here, $\mathrm{k}$ is the usual wave vector $\left\lfloor\frac{2 \pi}{\lambda}\right\rfloor(1$ is the impact factor [either zero or one] depending on the collision direction and $\delta_{1}(\mathrm{k})$ is a modules called the partial wave amplitude the M.T.C.S(Mixed Tabulated Complex Series. on the other hand is raised as the result of different impact angle and it is defined as the cross section produced through momentum exchange between the colliding species defined by[5]

$\sigma_{M T}=\frac{4 \pi}{k} \operatorname{Im} f(k, \theta=0) \ldots$

Where $I m$ is the imaginary part of the complex wave amplitude.

Analysis of equation (1) and (2) will be through lengthily [but straight forward 
Bessel function of the first kind [6] and using Matlabk2 program the results are

$$
\sigma_{e x}=\frac{4 \pi}{K^{2}}\left[\tan ^{-1} \frac{(K a)^{(2 l+1)}}{(2 l+1)(2 l-1) !}\right] \ldots
$$

Here (a) is a constant depending on the energy of excitation.

Finally the M.T.C.S will be manipulated and using the same category as for $\sigma_{e x}$ ,we obtain:

$$
\begin{aligned}
& \sigma_{M T}=\frac{4 \pi}{K^{2}}\left[1-\frac{K^{2}}{(1-2 l)}+\frac{\left(\frac{k^{2}}{2}\right)^{2}}{2 !(1-2 l)(3-2 l)}-\right. \\
& \left.\frac{\left(\frac{k^{2}}{2}\right)^{3}}{3 !(1-2 l)(3-2 l)(5-2 l)}--\right] \ldots(4)
\end{aligned}
$$

The values of these cross sections are shown in Figures (2),(3) and (4) respectively.

Stimulated Emission cross section is defined through the analogy of absorption cross section on which is the rate of absorption of energy per atom[6]. $\sigma_{a b}=\frac{4 \pi^{2} \alpha h^{2}}{m v}|\operatorname{Re} f(c k)| \ldots$. (5)

But the stimulated emission cross section is deferent in mechanism[7].

In gas lasers $\sigma_{s t}$ is formulated through the atomic transition characteristics.

In fact, it's the product of population inverse difference and the popular radiation width

$\rho(v)$

$=\left[\frac{c^{2}}{8 \pi v^{2}} A_{12}(v)\right]$

The 1 st one is $\left[N_{2}-N_{1}\right] \frac{g_{2}}{g_{1}}$ and $\mathrm{A}_{21}$ is called Einstein coefficient for spontaneous emission, from this definition[7].

$\sigma=\frac{4 \pi^{2 \alpha} h^{2}}{m v} \lambda_{21} A_{21}\left[N_{2}-N_{1}\right] \frac{g_{2}}{g_{1}} \ldots$

$\alpha$ is constant called the fine structure constant: $\frac{1}{(137)^{2}}, \lambda$ is the wavelength of radiation $\hbar$ is $\left(\frac{h}{2 \pi}\right)$ the famous Planks constant, $g_{1}$ and $g_{2}$ are called the statistical weight of the upper and lower atomic state $(2 \mathrm{~J}+1)$ were $\mathrm{J}$ is total angular momentum $\mathrm{m}$ is the mass of atomic contributing

species (here is Xenon) since Neon is just activating material [8].
The energy level diagram of the mixture is shown in figure number(1).

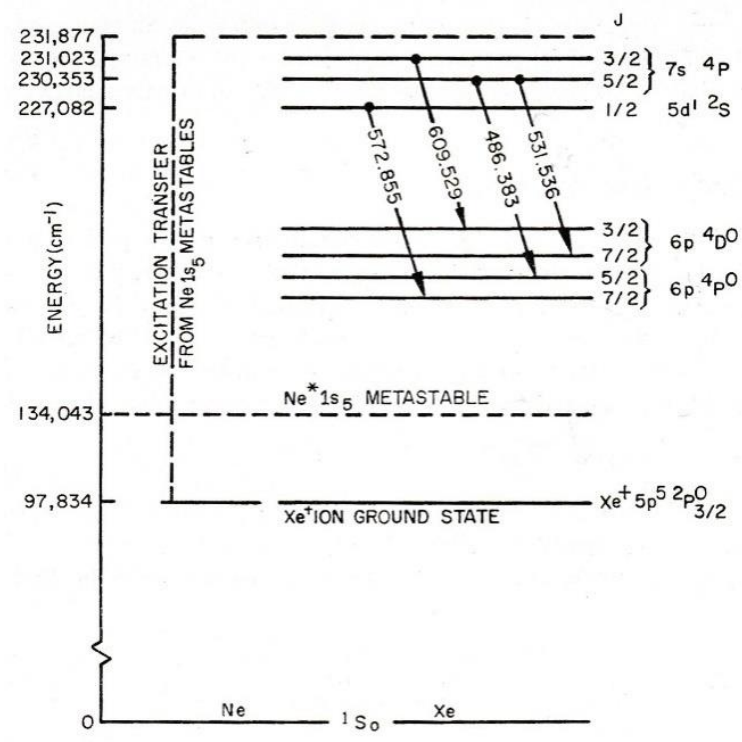

Fig.(1):Energy level diagram for Xenon and Neon gases[2]

\section{Results and Discussion:}

A mixture has been used with the following properties throughout the whole work, we tried to overcome all problems concerning different excitation processes facing a mixture of xenon and neon gases by the ratio of 30:70[ Xe:Ne] in a discharge tube hopping that it will be used as an active medium for an ion laser known as Xenon-Neon ion laser.

6) The parameters are important to be considered and they are the ionization potentials of these gases as $12.13 \mathrm{eV}$ and $21.56 \mathrm{eV}$ as in Figure(1)respectively. These two values give the idea that $\mathrm{Xe}$ ionizes (9.43)eV before its counterpart Neon, so the resonant transfers for Xenon from Neon at this value gives the possibility of $(\mathrm{Xe})$ to have almost many excited states after ionization is well known that two main processes[atomic and ionic] are in question:

a. The excitation cross - section for both gases in the range of $(0-20) \mathrm{eV}$ for $\mathrm{Xe}$, and (0-50)eV for Ne respectively.

The results of these calculations are shown in Figure(2).

b. Avery popular and famous counter part of the first one is the 
momentum transfer cross - section with in the range mentioned earlier, the results of these variations are shown in Figure (3)for the mixture fixed as (30:70) percent for $(\mathrm{Xe}: \mathrm{Ne})$ gases.

B.Bransden and Joachain[9] have proposed the ionization cross - section for both gases at their peak values to be $\left(0.113 \times 10^{-16}\right) \mathrm{cm}^{2}$ for Xenon and ( $\left.0.0333 \times 10^{-16}\right) \mathrm{cm}^{2}$ for neon respectively. Our values in Figure(5) give them a $\left(0.11 \times 10^{-16}\right.$ and $\left.0.032 \times 10^{-16}\right) \mathrm{cm}^{2}$ for the gases which are in good agreement with the experimental values described.

c. Stimulated emission cross-section found both theoretically and experimentally give what is called [saturation output intensity if they are used as an active medium for a medium powered Ion laser workable at (5371 ) was:

$I_{\text {sat }}=\frac{h v_{L}}{\tau_{m \sigma_{s t}}}$

Her $v_{\mathrm{L}}$ is the frequency of the operating laser wave length. $\tau_{m}$ is the spontaneous life time at the $5 \mathrm{p}$ state and $\sigma_{\mathrm{st}}$ is the desired stimulated emission crosssection.

Here $v_{\mathrm{L}}=\mathrm{c} / \lambda=\frac{3 \times 10^{8}}{0.5371 \times 10^{-6}}=5.58 \times$ $10^{14} \mathrm{~Hz}$.

$\tau_{\mathrm{s}}=8.2 \mathrm{nsec} . \sigma_{\mathrm{st}}=0.0333 \times 10^{-20} \mathrm{~m}^{2}$.

As Such:

$\mathrm{I}_{\mathrm{sat}}=\frac{6.625 \times 10^{-34} \times 5.58 \times 10^{14}}{8.2 \times 10^{-9} \times 0.0333 \times 10^{-20}}=135.38 \frac{\mathrm{w}}{\mathrm{m}^{2}}$.

The theory of Boltzmann transverse mixtures predicated that at these circumstances, Inert gases are to be considered as cold-plasma and Enhanced by Electrical methods.

d. Finally, much work have been done to find out the proposed stimulation emission cross - section for these gases in their peak positions since, in laser, this parameter plays an important role for the output power they were $\left(0.3 \times 10^{-}\right.$ $\left.{ }^{16}\right) \mathrm{cm}^{2}$ and $\left(24 \times 10^{-16}\right) \mathrm{cm}^{2}$ for $\mathrm{Ne}$ and $\mathrm{Xe}$ respectively.
Now, recommended tool to be confident about the results is that we have to ascertain about the results of figure (4) which represents the sum of stimulated and ionization cross - section taking the values of pure ionization potential from this result, we obtained $\left(0.1333 \times 10^{-}\right.$ $\left.{ }^{16}\right) \mathrm{cm}^{2}$ for the mixture which is quite reasonable with the value obtained by [9] to be $\left(0.1323 \times 10^{-16}\right) \mathrm{cm}^{2}$.

\section{Conclusion:}

In this study the tendency was towards a formulation for what is called stimulated emission cross-section in a mixture containing Xenon and Neon gases. The test was through fixing a ratio which is common in the field of cold plasma parameter calculations. The facts obtained in the process are:

The best fitting ratio for manipulating the mixture thermodynamically was (Xenon: Neon) as (30:70) as usual. This ratio is coming from the fact that at this ratio, the resonance transition between $\mathrm{Ne} 2 p$ and $\mathrm{Xe} 5 \mathrm{p}$ are in its highest activity.

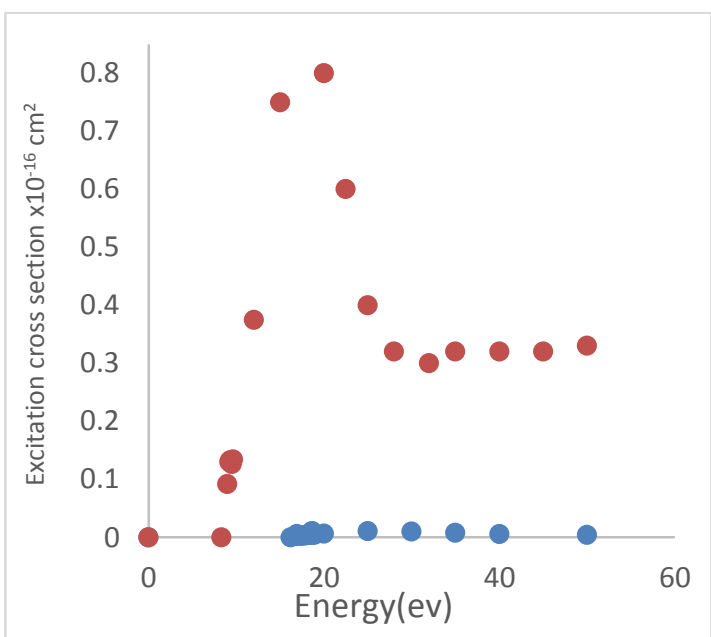

Fig.[2].Excitation cross section for Xenon and Neon as a function of energy 


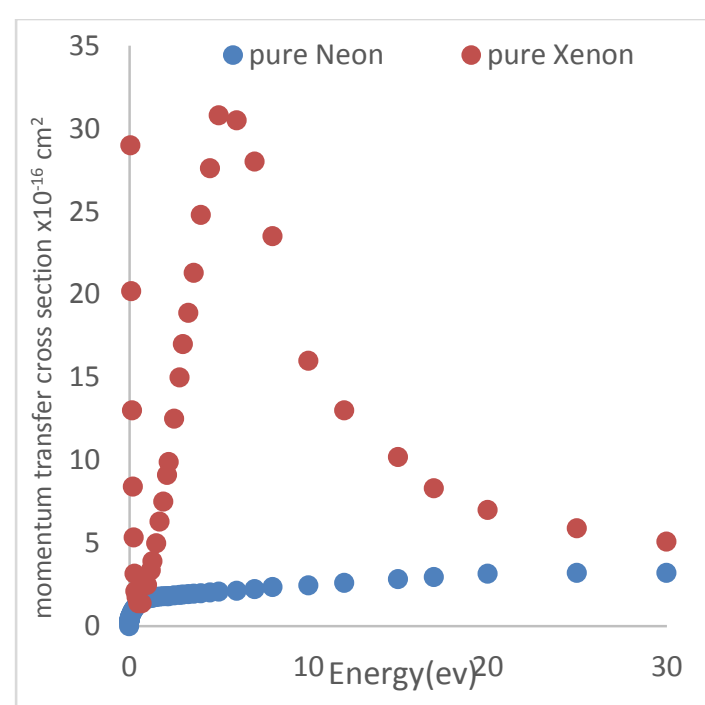

Fig.[3].Variation of momentum transfer cross section as a function of energy for each of Xe and Ne.

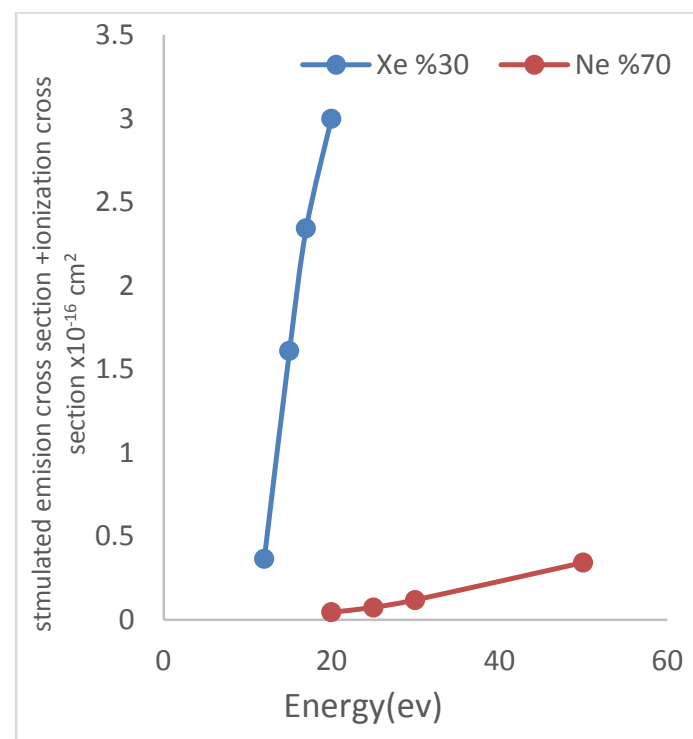

Fig[4].combination of stimulated emission cross section +ionization cross section for xenon and neon gases as a function of energy.

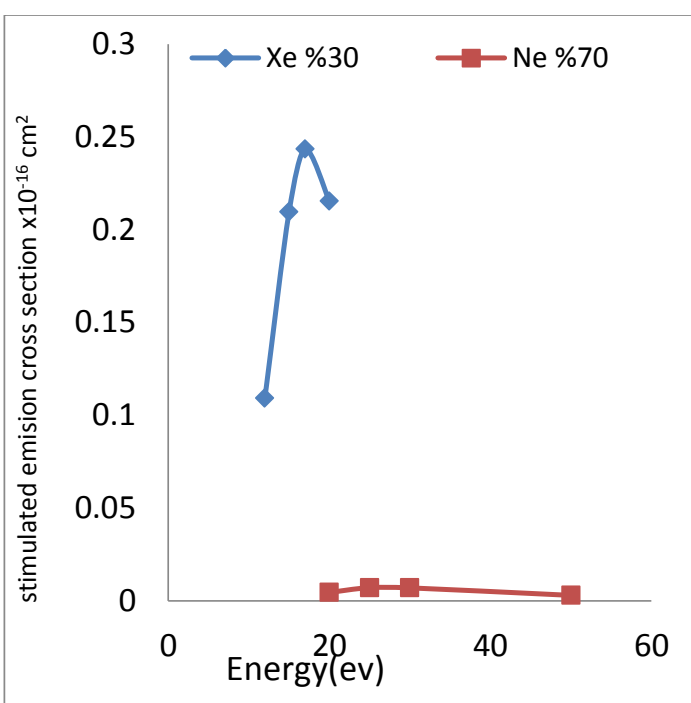

Fig.[5].stimulated emission cross section for Xenon Neon gas mixture as a function of energy.

\section{References:}

[1] Hasted, J. B. and Phil, M. A. D. 1964. Physics of Atomic collisions, London Butterworth's.

[2] Colin, S.; Willett, 1984. Introduction to gas lasers: Population inversion Mechanisms, Ph. D., M. Inst. P. vol.67, Oxford. New York. Toronto. Sydney.

[3] Hayashi, M. 2003. Determination of Electron and excitation crosssections, Journal of Applied Physics, 26:(581-589).

[4] Machacek, J. R. and Jone, A. C. 2011. Journal of Physics [Low energy interaction with xenon], (13):19-34.

[5] Meunier, J. and Boeuf, J. P. 1995. Numerical model of ac plasma display panel cell in neon-xenon mixtures, J. Appl. Phys 78,2: 731745.

[6] MOTT.N.F and MASSEY.H.S.W, 1965, The Theory of Atomic Collisions, Oxford University Press.

[7] Brovik, A.; Rausch, J. and Muller, A. Electron Impact Ionization of Xenon, 2014. XXXVI International conference of photonic, electron and (IOP) Atomic collisions,(2426)/6/2014, SE SAME, JORDAN. 
[8] Heylen, A. E. 1999. The relationship between electronmolecule collision cross-sections, experimental Townsend primary and secondary ionization coefficients and constants, electric strength and molecular structure of

gaseous hydrocarbons, Physics of ionized gases, p3005.

[9] Bransden, B. and Joachain, E. 2004. Fundamentals of light sources at lasers, Mark Csele Willey inter science

$$
\begin{aligned}
& \text { مساحة مقطع الاتبعاث المحفز فى الليزر نيون - زينون } \\
& \text { كَولّالّه ياسين بكر } \\
& \text { قسم الفيزياء ، كلية التربية ، جامعة صلاح الدين، صلاح الدين، العراق. }
\end{aligned}
$$

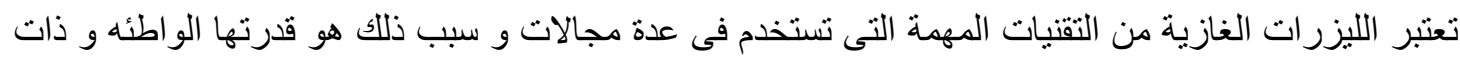

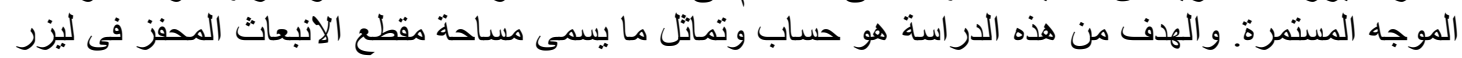

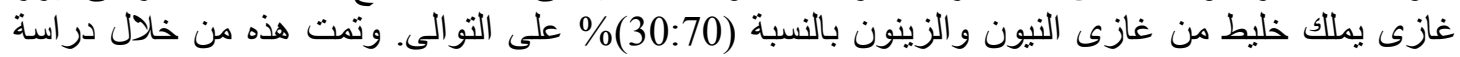
نظرية لكل غاز على حدة من حيث الصفات الفيزيائية تعمل كوسط فعال وكانت النتائج منطقية و اكثر مناسبة من

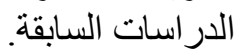

$$
\text { الكلمات المفتاحية: خليط الغاز ات، عمليات التاين و التهيج، الليزرات الغازية. }
$$

